\title{
MIGRAÇÃO PSPI BASEADA NAS EQUAÇÕES DA ONDA UNIDIRECIONAIS COM AMPLITUDE VERDADEIRA
}

\author{
Flor A. Vivas ${ }^{1}$ e Reynam C. Pestana ${ }^{2}$ \\ Recebido em 26 outubro, 2006 / Aceito em 15 março, 2007 \\ Received on October 26, 2006 / Accepted on March 15, 2007
}

\begin{abstract}
The true-amplitude one-way wave equation suggested recently is both dynamically and kinematically correct. This one-way wave equations produces accurate traveltime in accordance to the eikonal equation and accurate amplitudes in accordance to the transport equation for the two-way wave equation. In laterally inhomogeneous media the implementation of this true-amplitude one-way wave equation involves the numeric calculation of a differential-integral operator that can be approximated by summation and solved by finite-difference methods. In only depth-dependent media, the new term in the one-way wave equation can be eliminated and the new one-way wave equations can be solved using the conventional phase-shift method with the appropriated boundary and imaging condition, reduced to products in the Fourier domain. Here, we present an extension of phase shitt plus interpolation (PSPI) migration for true-amplitude migration based in the phase-shift true-amplitude migration developed in depth-dependent media. Numerical tests in the common shot domain with the new PSPI true-amplitude method and the Extended split-step PSPI-SS proposed were compared with the output of the conventional PSPI and PSPI-SS. The imaging quality in relation to amplitude is better with the proposed methods and the kinematic part is preserved.
\end{abstract}

Keywords: common-shot migration, true-amplitude migration, one-way wave equations, depth migration, PSPI migration, PSPI-SS migration.

RESUMO. As equações da onda unidirecionais com amplitude verdadeira, propostas nos trabalhos mais recentes, levam em conta de forma correta a parte cinemática e dinâmica da equação completa da onda equivalentemente às obtidas a partir da aproximação de altas freqüências. Isto é, as equações iconal e de transporte obtidas para estes operadores se correspondem com as obtidas com a equação completa da onda. Num meio com variação lateral de velocidade, a implementação das equações unidirecionais com amplitude verdadeira envolve o cálculo numérico de operadores integro-diferenciais que podem ser aproximados por somatórios e resolvidos por técnicas de diferenças finitas. Para um meio que varia somente com a profundidade, as novas equações unidirecionais com amplitude verdadeira podem ser solucionadas através do tradicional método phase-shift, e as novas condições de fronteira e de imagem se reduzem a multiplicações no domínio de Fourier. Neste trabalho estamos propondo estender a migração phase-shift com amplitude verdadeira para meios com variação lateral $v(x, z)$, através da metodologia Phase shift plus interpolation (PSPI). Experimentos numéricos no domínio de tiro comum com o novo método PSPI com amplitude verdadeira e sua extensão split-step (PSPI-SS), produzem imagens com amplitudes mais corretas em relação aos operadores unidirecionais convencionais, enquanto as características cinemáticas da migração PSPI e PSPI-SS são preservadas.

Palavras-chave: migração de tiro comum, migração com amplitude verdadeira, equações unidirecionais, migração em profundidade, migração PSPI, migração PSPI-SS.

\footnotetext{
${ }_{1}^{1}$ Curso de Pós-graduação em Geofísica, Universidade Federal da Bahia, Campus Universitário da Federação, 40170-290 Salvador, BA, Brasil. Tel.: (71) 3283-8531; Fax: (71) 3283-8551-E-mail: favm@cpgg.ufba.br

${ }^{2}$ Departamento de Física da Terra e do Meio Ambiente, Instituto de Física/CPGG, Universidade Federal da Bahia, Campus Universitário da Federação, 40170-290 Salvador, BA, Brasil. Tel.: (71) 3283-8521; Fax: (71) 3283-8551-E-mail: reynam@cpgg.ufba.br
} 


\section{INTRODUÇÃo}

Em meios homogêneos as soluções da equação de onda são equivalentes às soluções de dois operadores de primeira ordem conhecidos como operadores unidirecionais da onda (One-Way Wave Equation - OWWE), cujos campos de onda correspondem aos campos que se propagam na direção ascendente ou descendente. 0 imageamento sísmico com os operadores unidirecionais é formulado como sendo a continuação do campo da fonte através da equação unidirecional para campos descendentes e a continuação do campo registrado nos receptores através da equação unidirecional para campos ascendentes, junto com um princípio de imageamento (Claerbout, 1971).

Em meios com variação lateral da velocidade, as equações unidirecionais não são mais válidas, dado que a transformada de Fourier espacial da equação de onda envolve neste caso uma convolução. Entretanto, existe um conjunto de técnicas (Gazdag \& Sguazzero, 1984; Claerbout, 1985; Stoffa et al., 1990; Ristow \& Rühl, 1994) que permite levar em conta, de forma aproximada, as variações laterais de velocidade, supondo que as equações unidirecionais continuam sendo válidas. Estas técnicas são utilizadas com êxito no imageamento sísmico em profundidade, no entanto as informaç̃̃es de amplitude não são consideradas de forma correta, isto é, os operadores unidirecionais são cinematicamente, mas não dinamicamente, corretos.

Zhang (1993) propôs incluir um novo termo nos operadores unidirecionais e mostrou que os novos operadores produzem as mesmas equações iconal e de transporte, que as obtidas para a equação de onda de duas vias. Zhang et al. (2001) mostraram que num meio homogêneo a modificação das condições de fronteira é necessária para obter um mapa dos reflectores com as mesmas amplitudes que se obtém através da migração/inversão Kirchhoff (Bleistein, 1987).

Em Zhang et al. (2005) se formula a migração de tiro comum baseada nos operadores unidirecionais com amplitude verdadeira para meios heterogêneos, e é apresentada uma implementação para meios $v(x, y, z)$ envolvendo o cálculo numérico de operadores integro-diferenciais que são aproximados por somatórios e resolvidos por técnica de diferenças finitas.

Neste trabalho apresentamos os novos operadores unidirecionais, junto com as condições de fronteira e de imagem propostas em Zhang et al. (2005), mostrando como no caso $v(z)$ o novo termo presente nos operadores pode ser eliminado e as novas equações podem ser solucionadas mediante 0 convencional esquema phase-shift. Apresentamos exemplos numéricos 2D, mostrando que no caso $v(z)$ as amplitudes são recuperadas de forma correta.
Finalmente estendemos 0 esquema de migração phase-shift com amplitude verdadeira para meios com variação lateral da velocidade $v(x, y, z)$, através das técnicas PSPI e PSPI-SS, sendo que o último método melhora o cálculo da fase aplicando a correção split-step para cada velocidade de referência (Kessinger, 1992; Aldunate et al., 2004).

Experimentos numéricos 2D mostraram melhoramento nas amplitudes da imagem migrada assim como preservação da parte cinemática nos dois métodos propostos.

\section{MIGRAÇÃO EM PROFUNDIDADE NO DOMÍNIO DO TIRO}

A migração em profundidade por continuação do campo de onda é feita em duas etapas; na primeira etapa os campos são continuados do nível de extrapolação $z$ ao nível $z+\Delta z$; na segunda etapa uma condição de imagem é aplicada. 0 domínio de migração pode ser de tiro comum ou fonte-receptor, sendo a migração de tiro comum a mais natural pelo fato de tratar cada tiro como um experimento físico independente e migrar estes separadamente. A seção migrada final é obtida somando-se os tiros migrados (Schultz \& Sherwood, 1980).

A migração de cada tiro $S$ é feita mediante o modelamento do campo de onda da fonte, como um campo descendente $D_{s}$, e a depropagação do campo registrado nos receptores, como um campo ascendente $U_{s}$, através dos operadores unidirecionais

$$
\begin{gathered}
{\left[\frac{\partial}{\partial z}+i \frac{\omega}{v} \sqrt{1+\frac{v^{2}}{\omega^{2}}\left(\frac{\partial^{2}}{\partial x^{2}}+\frac{\partial^{2}}{\partial y^{2}}\right)}\right] D_{s}=0,} \\
D_{s}(x, y, z=0 ; \omega)=\delta\left(x-x_{s}, y-y_{s}, z\right), \\
{\left[\frac{\partial}{\partial z}-i \frac{\omega}{v} \sqrt{1+\frac{v^{2}}{\omega^{2}}\left(\frac{\partial^{2}}{\partial x^{2}}+\frac{\partial^{2}}{\partial y^{2}}\right)}\right] U_{s}=0,} \\
U_{S}(x, y, z=0 ; \omega)=Q(x, y ; \omega) .
\end{gathered}
$$

Segundo o princípio de imageamento de Claerbout (1971) "O refletor existe nos pontos da subsuperfície onde a primeira chegada da onda descendente coincide no tempo $\left(t_{D}\right)$ e no espaço $(x, y, z)$ com a onda ascendente". Esta condição é expressa matematicamente como segue:

$$
R_{S}(x, y, z)=\frac{U_{S}\left(x, y, z, t_{D}\right)}{D_{S}\left(x, y, z, t_{D}\right)},
$$

mas como não se conhece, a priori, o tempo $t_{D}$ em (3), precisase de um esquema prático para localizar a posição do refletor e estimar um valor de amplitude proporcional à refletividade. 
Claerbout (1971) mostra que a amplitude e a posição do refletor correspondem ao "lag" zero da correlação cruzada na dimensão temporal dos campos de onda descendente e ascendente, o que equivale no domínio da freqüência a

$$
\begin{aligned}
R_{S}(x, y, z) & =\int \frac{U_{S}(x, y, z, \omega)}{D_{S}(x, y, z, \omega)} d \omega \\
& =\int \frac{U_{S}(x, y, z, \omega) \bar{D}_{S}(x, y, z, \omega)}{D_{S}(x, y, z, \omega) \bar{D}_{S}(x, y, z, \omega)} d \omega .
\end{aligned}
$$

Esta condição de imagem é conhecida como condição de imagem por deconvolução, pois um processo de deconvolução está implícito em (4).

Dado que 0 espectro de $D_{s}(x, y, z, \omega) \bar{D}_{s}(x, y, z, \omega)$ é real e, portanto, não contém informações de fase, uma implementação natural da condição de imagem sem levar em conta a amplitude omite 0 denominador em (4), evitando divisão por pequenas quantidades e portanto imagens muito ruidosas, e é dado por:

$$
R_{S}(x, y, z)=\int U_{s}(x, y, z, \omega) \bar{D}_{s}(x, y, z, \omega) d \omega,
$$

que é conhecida como condição de imagem por correlação.

A condição de imagem por correlação (5), embora tenha a implementação mais simples e robusta, apresenta problemas na reconstrução de amplitudes para estimar refletividades por falta da normalização com a amplitude do campo descendente.

Uma outra alternativa é escrever a condição de imagem (4) na forma

$$
\begin{gathered}
R_{S}(x, y, z)= \\
\sum_{\omega} \frac{U_{s}(x, y, z, \omega) \bar{D}_{s}(x, y, z, \omega)}{D_{S}(x, y, z, \omega) \bar{D}_{s}(x, y, z, \omega)+\epsilon^{2}},
\end{gathered}
$$

onde 0 termo $\epsilon$ é uma constante ou uma função que depende de $(x, y, z) .0$ termo $\epsilon$ pode ser calculado como (Valenciano \& Biondi, 2003)

$$
\epsilon^{2}(x, y, z)=\lambda \sum_{\omega} D_{S}(x, y, z, \omega) \bar{D}_{s}(x, y, z, \omega),
$$

onde $\lambda$ é uma constante cujo valor vai depender do dado.

Observe-se que nos pontos $(x, y, z)$, onde a amplitude do campo descendente é muito pequena para qualquer $\omega$, a estabilização através de (7) não tem efeito nenhum, continuando a imagem a ter muito ruído.

Neste trabalho todas as imagens são obtidas através da técnica proposta por Guitton et al. (2006), onde é feita uma filtragem do espectro de amplitude de $D_{s}(x, y, z, \omega) \bar{D}_{s}(x, y, z, \omega)$, antes de aplicar a condição de imagem (4). A suavização espacial do espectro de amplitude é feita para cada frequêencia, preenchendo os zeros espectrais com valores calculados a partir dos valores na vizinhança. A condição de imagem suavizada fica

$$
\begin{gathered}
R_{S}(x, y, z)= \\
\int \frac{U_{S}(x, y, z, \omega) \bar{D}_{s}(x, y, z, \omega)}{\ll D_{S}(x, y, z, \omega) \bar{D}_{S}(x, y, z, \omega) \gg} d \omega,
\end{gathered}
$$

onde $\ll \gg$ representa a suavização espacial. Para 0 caso 2D implementamos uma suavização na direção $x$ com um filtro triangular, 0 que equivale a uma suavização com filtro retangular feita duas vezes. Nesta condição de imagem o parâmetro a ser escolhido é o número de amostras do filtro 0 qual vai depender do tamanho e complexidade do campo de velocidades.

\section{EQUAÇÕES DE ONDA UNIDIRECIONAIS COM AMPLITUDE VERDADEIRA}

Zhang (1993) propõe incluir um novo termo nos operadores unidirecionais, equações (1) e (2), e mostra mediante análise assintótica de altas freqüências que as novas equações, junto com uma nova condição de imagem, proporcionam as mesmas amplitudes que as obtidas mediante um processo de migração/inversão Kirchhoff. Além de incluir o novo termo, a condição inicial do campo descendente deve ser modificada, como proposto por Wapenaar (1990), e assim modelar de forma correta uma fonte de injeção de volume com as equações unidirecionais.

Em Zhang (2005) um esquema de migração para meios $v(x, y, z)$ é formulado baseado nas seguintes equações

$$
\begin{gathered}
\left(\frac{\partial}{\partial z}+i \Lambda-\Gamma\right) p_{D}=0 \\
p_{D}(x, y, z=0 ; \omega)=\frac{1}{2 i \Lambda} \delta\left(x-x_{s}, y-y_{s}, z\right), \\
\left(\frac{\partial}{\partial z}-i \Lambda-\Gamma\right) p_{U}=0 \\
p_{U}(x, y, z=0 ; \omega)=Q(x, y ; \omega),
\end{gathered}
$$

com os operadores

$$
\begin{gathered}
\Lambda=\frac{\omega}{v} \sqrt{1+\frac{\Delta_{T}}{\omega^{2}}}, \\
\Gamma=\frac{v_{z}}{2 v}\left[1-\left(\omega^{2}+\Delta_{T}\right)^{-1} \Delta_{T}\right],
\end{gathered}
$$


onde

$$
v_{z}=\frac{\partial v(x, y, z)}{\partial z}
$$

$$
\Delta_{T}=\left(v \frac{\partial}{\partial x}\right)^{2}+\left(v \frac{\partial}{\partial y}\right)^{2}
$$

A condição de imagem é dada por

$$
R(x, y, z)=\frac{1}{2 \pi} \int \frac{p_{U}(x, y, z ; \omega)}{p_{D}(x, y, z ; \omega)} d \omega,
$$

onde os campos $p_{D}$ e $p_{U}$ estão relacionados com $D$ e $U$ através das equações

$$
\left\{\begin{array}{l}
p_{D}=(i \Lambda)^{-1} D \\
p_{U}=(i \Lambda)^{-1} U
\end{array}\right.
$$

\section{MIGRAÇÃO OWWE COM AMPLITUDE VERDADEIRA EM MEIOS $v(z)$}

Num meio em que a velocidade de propagação varia apenas verticalmente, podemos aplicar transformada de Fourier nas variáveis transversais $x, y$ e escrever as equações unidirecionais de amplitude verdadeira (9)-(12) de forma explícita como:

$$
\begin{gathered}
\left(\frac{\partial}{\partial z}+i \lambda-\gamma\right) p_{D}=0, \\
p_{D}\left(k_{x}, k_{y}, z=0 ; \omega\right)=\frac{1}{2 i \lambda} e^{i\left(k_{x} x_{s}+k_{y} y_{s}\right)}, \\
\left(\frac{\partial}{\partial z}-i \lambda-\gamma\right) p_{U}=0, \\
p_{U}\left(k_{x}, k_{y}, z=0 ; \omega\right)=Q\left(k_{x}, k_{y} ; \omega\right),
\end{gathered}
$$

com os operadores

$$
\begin{gathered}
\lambda=\frac{\omega}{v} \sqrt{1-\frac{v^{2}}{\omega^{2}}\left(k_{x}^{2}+k_{y}^{2}\right)} \\
\gamma=\frac{v_{z}}{2 v} \frac{\omega^{2}}{\omega^{2}-v^{2}\left(k_{x}^{2}+k_{y}^{2}\right)}=\frac{\partial}{\partial z}\left(\ln \frac{1}{\sqrt{\lambda}}\right) .
\end{gathered}
$$

É fácil verificar que introduzindo novos campos de onda $q_{D}$ e $q_{U}$ na forma

$$
\left\{\begin{array}{l}
q_{D}=(\lambda)^{1 / 2} p_{D} \\
q_{U}=(\lambda)^{1 / 2} p_{U}
\end{array}\right.
$$

as equações (15) e (16) se reduzem a

$$
\left(\frac{\partial}{\partial z}+i \lambda\right) q_{D}=0
$$

$$
q_{D}\left(k_{x}, k_{y}, z=0 ; \omega\right)=\frac{1}{2 i \lambda^{1 / 2}} e^{i\left(k_{x} x_{s}+k_{y} y_{s}\right)},
$$

$$
\left(\frac{\partial}{\partial z}-i \lambda\right) q_{U}=0,
$$

$$
q_{U}\left(k_{x}, k_{y}, z=0 ; \omega\right)=\lambda^{1 / 2} Q\left(k_{x}, k_{y} ; \omega\right) .
$$

Assim, 0 algoritmo de migração phase-shift convencional pode ser implementado para migração num meio $v(z)$ com amplitude verdadeira, através das equações (20) e (21) com as seguintes etapas:

- Transformada de Fourier da fonte e dos dados registrados para 0 domínio $\left(k_{x}, k_{y} ; \omega\right)$.

- Nível $z=0$, modificação da condição de fronteira na fonte pelo fator $\frac{1}{2 i \lambda^{1 / 2}}$.

- Nível $z=0$, modificação da condição de fronteira dos dados registrados nos receptores pelo fator $\lambda^{1 / 2}$.

- Extrapolação em profundidade dos campos $q_{D}$ e $q_{U}$, equações (20) e (21), através do método de phase-shift.

- Obtenção dos campos $p_{D}$ e $p_{U}$ através das relações (19) em cada nível em profundidade.

- Transformada de Fourier inversa para o domínio $(x, y ; \omega)$ dos campos $p_{D}$ e $p_{U}$.

- Condição de imagem (13) em cada nível em profundidade.

\section{MIGRAÇÃO PSPI E PSPI-SS COM AMPLITUDE VERDADEIRA}

Em meios com variação lateral de velocidade a implementação das equações unidirecionais com amplitude verdadeira é mais complexa pelo fato de ter envolvido os operadores pseudodiferenciais $\Lambda$ e $\Gamma$, dadas pelas equações (11) e (12). Em Zhang (2005) se utiliza uma expressão integro-diferencial para $\Lambda$ e são introduzidas as variáveis $q_{D}$ e $q_{U}$ a fim de eliminar 0 termo $\Gamma$, da mesma forma que se fez para um meio que varia com a profundidade $v(z)$. 0 operador pseudo-diferencial $\Lambda$ é aproximado por uma soma e resolvido por métodos de diferenças finitas.

Como uma outra alternativa, propomos incluir as variações laterais de velocidade através do esquema PSPI baseado no algoritmo phase-shift de amplitude verdadeira para um meio $v(z)$. 
0 esquema PSPI trabalha no domínio misto $(\omega-k / \omega-x)$, fazendo a primeira mudança de fase no domínio $(\omega-k)$ através do método phase-shift para várias velocidades de referência. As variações laterais de velocidade são introduzidas no domínio $(\omega-x)$ interpolando-se os campos obtidos por phase-shift para as diferentes velocidades de referência segundo o valor da velocidade local.

Para 0 caso de duas velocidades de referência $v_{1} \mathrm{e} v_{2}$, em cada nível $z$, com $v_{2}>v_{1}$, tem-se

$$
\begin{gathered}
P(x, z+\Delta z, \omega)= \\
\alpha P_{1}(x, z+\Delta z, \omega)+(1-\alpha) P_{2}(x, z+\Delta z, \omega),
\end{gathered}
$$

onde

$$
\alpha=\frac{v_{2}-v(x)}{v_{2}-v_{1}} .
$$

Não existe um único critério para a seleção do número e valores das velocidades de referência, dado que existe uma dependência das características do campo de velocidades. Neste trabalho utiliza-se 0 critério de entropia como medida da dispersão das velocidades (Bagaini et al., 1995), para determinar 0 número e as velocidades de referência ótimas em cada nível de extrapolação.

Desde que nos esquemas de migração PSPI a interpolação é feita com os campos de onda obtidos para as diferentes velocidades de referência, espera-se que a informação de amplitude seja interpolada de forma tão correta quanto a informação da fase.

0 algoritmo de migração PSPI com amplitude verdadeira é implementado mediante as seguintes etapas:

- Transformada de Fourier da fonte e dos dados registrados para o domínio $\left(k_{x}, k_{y} ; \omega\right)$.

- Nível $z=0$, modificação da condição de fronteira na fonte pelo fator $\frac{1}{2 i \lambda^{1 / 2}}$.

- Nível $z=0$, modificação da condição de fronteira dos dados registrados nos receptores pelo fator $\lambda^{1 / 2}$.

- Seleção do número de velocidades de referência ótimas em cada nível de profundidade.

- Extrapolação em profundidade pelo método phase-shift dos campos $q_{D}$ e $q_{U}$ com as diferentes velocidades de referência.

- Obtenção dos campos $p_{D}$ e $p_{U}$ através das relações (19) em cada nível em profundidade.
- Transformada de Fourier inversa para o domínio $(x, y ; \omega)$ dos campos $q_{D}, q_{U}, p_{D}$ e $p_{U}$.

- Interpolação dos campos $q_{D}, q_{U}, p_{D}$ e $p_{U}$.

- Condição de imagem (13) em cada nível em profundidade.

0 custo computacional do método PSPI 2D convencional relacionado ao número de FFTs (Fast Fourier Transform) é de

$$
\begin{aligned}
N= & 2 \cdot\left(n_{t} \cdot \log n_{t}\right)+2 \cdot n_{z} \cdot\left(n_{x} \cdot \log n_{x}\right) \\
& +2 \cdot n_{z} \cdot n_{v_{z}} \cdot\left(n_{k_{x}} \cdot \log n_{k_{x}}\right),
\end{aligned}
$$

onde $n_{t}$ é o número de amostras no tempo, $n_{x}, n_{z}$ o número de amostras no campo de velocidades, $n_{k_{x}}$ o número de números de onda na direção $x$ e $n_{v_{z}}$ o número de velocidades de referência determinado em cada nível de extrapolação.

No método PSPI com amplitude verdadeira aqui proposto, tem-se um custo adicional relacionado com a introdução de campos diferentes para extrapolação $q_{D}, q_{U}$ e para imageamento $p_{D}, p_{U}, 0$ custo computacional do método PSPI com amplitude verdadeira fica expresso como

$$
\begin{aligned}
N= & 2 \cdot\left(n_{t} \cdot \log n_{t}\right)+2 \cdot n_{z} \cdot\left(n_{x} \cdot \log n_{x}\right) \\
& +4 \cdot n_{z} \cdot n_{v_{z}} \cdot\left(n_{k_{x}} \cdot \log n_{k_{x}}\right) .
\end{aligned}
$$

Uma melhor correção da fase é obtida mediante 0 método Extended Split-step (Kessinger, 1992), o qual foi implementado como um PSPI que inclui a correção de fase Split-step para cada velocidade de referência (Aldunate et al., 2004) e por isto é denominado PSPI com Split-step ou PSPI-SS.

\section{EXPERIMENTOS NUMÉRICOS}

Os dois algoritmos descritos, PSPI e PSPI-SS, foram implementados para dados 2D e a condição de imagem tipo deconvolução, com suavização do campo descendente através do filtro triangular, foi utilizada em todos os casos. Apresentamos em primeiro lugar os resultados da migração phase-shift com amplitude verdadeira para um meio que varia com a profundidade $v(z)$.

0 dado é gerado mediante modelamento tipo Kirchhoff do pacote Seismic Un*x (Cohen \& Stockwell, 2006), que contém um único tiro na posição zero e receptores em afastamentos de $-6 \mathrm{~km}$ a $6 \mathrm{~km}$, com espaçamento uniforme de $20 \mathrm{~m}$. 0 modelo contém quatro refletores horizontais nas profundidades $z=$ $1000 \mathrm{~m}, 2000 \mathrm{~m}, 3000 \mathrm{~m}, \mathrm{e} 4000 \mathrm{~m}$ e velocidade do meio $v(z)=$ $2000+0.3 z(\mathrm{~m} / \mathrm{s})$. Os quatro refletores modelam contrastes de densidade unitária e por isso o coeficiente de reflexão recuperado não deve depender nem do ângulo de reflexão, nem da profundidade do refletor. 

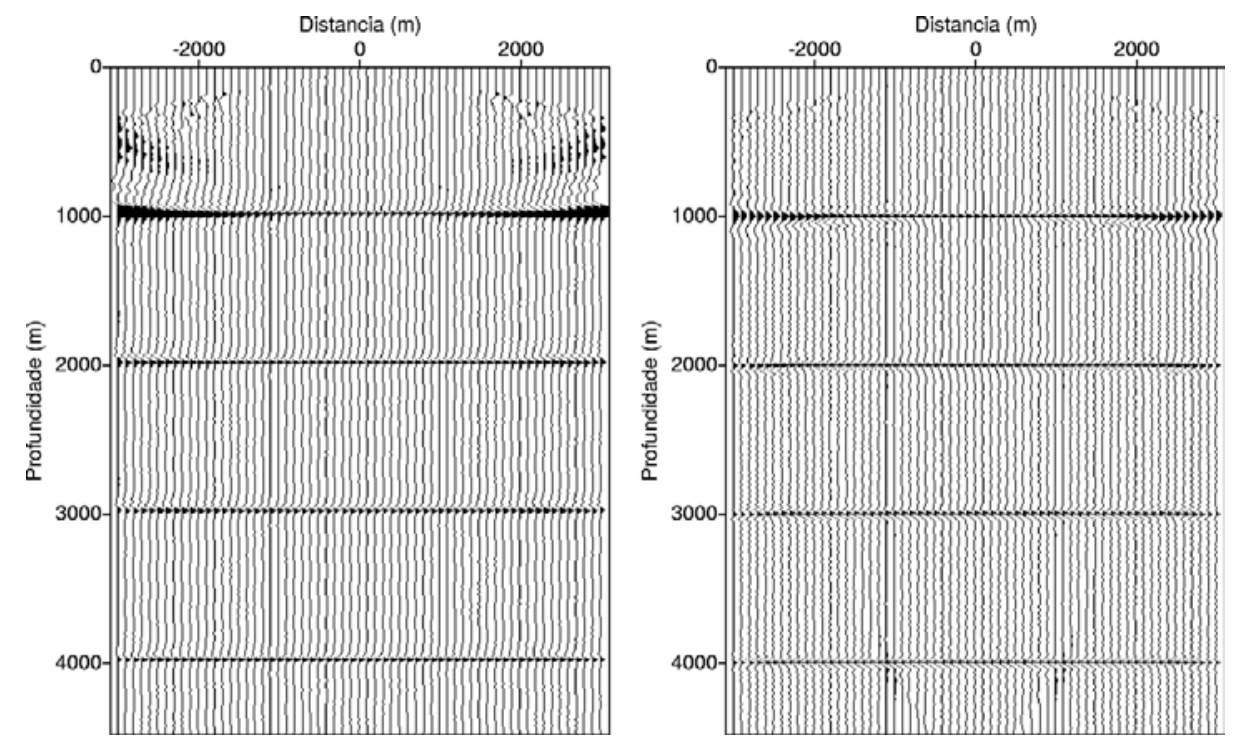

Figura 1 - Imagem obtida através do método phase-shift convencional (esquerda). Imagem através do método phase-shift com amplitude verdadeira (direita).

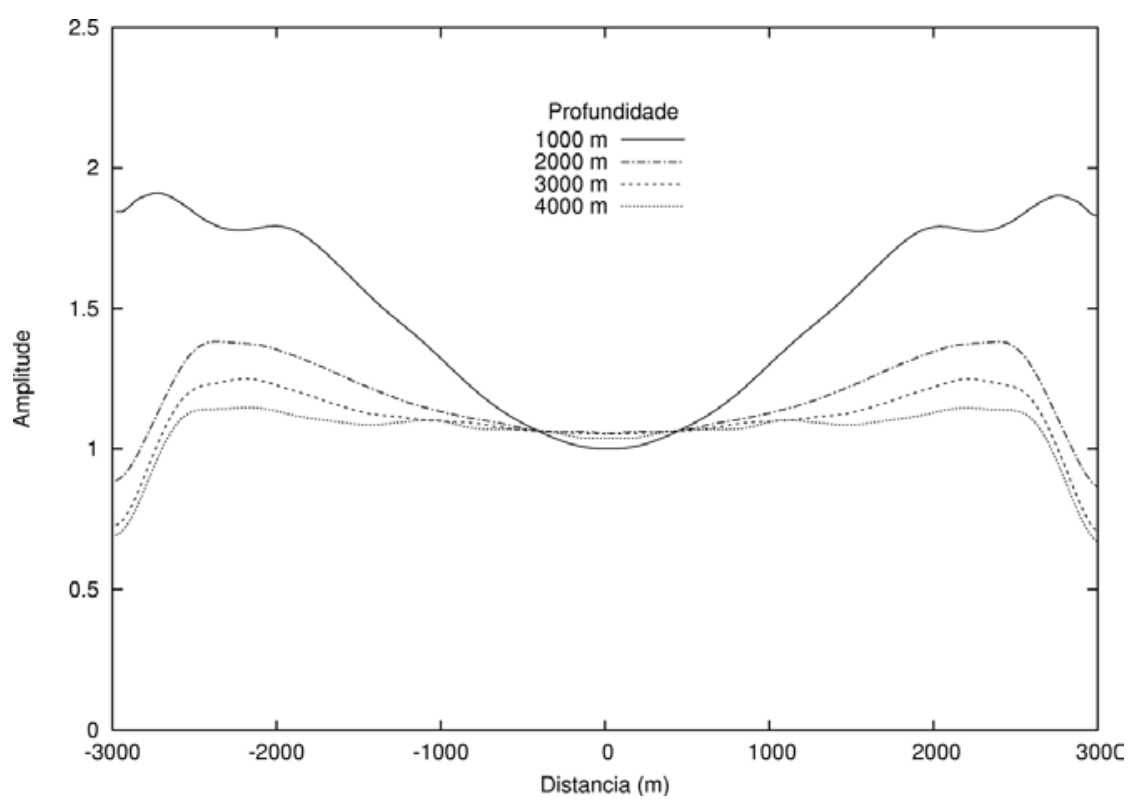

Figura 2 - Amplitudes nos refletores através da migração phase-shift convencional.

Na Figura 1 são mostradas as seções migradas com phaseshift convencional, à esquerda, e com phase-shift com amplitude verdadeira, à direita.

Nas Figuras 2 e 3 estão apresentadas as amplitudes obtidas sobre cada refletor com migração convencional e com migração com amplitude verdadeira, respectivamente. Observa-se que além da correção da amplitude em relação ao ângulo, obteve-se também correção da amplitude com a profundidade.
0 esquema de extrapolação phase-shift com amplitude verdadeira para meios $v(z)$ foi adaptado para a migração PSPI. No experimento numérico utilizou-se o mesmo modelo de quatro refletores horizontais com contrastes de densidade unitária, num meio com velocidade $v(x, z)=2000+0.2 x+0.3 z(\mathrm{~m} / \mathrm{s})$. $\mathrm{Na}$ Figura 4 apresenta-se 0 campo de velocidades de referência e na Figura 5 dado sintético.

A Figura 6 corresponde às imagens migradas com os 


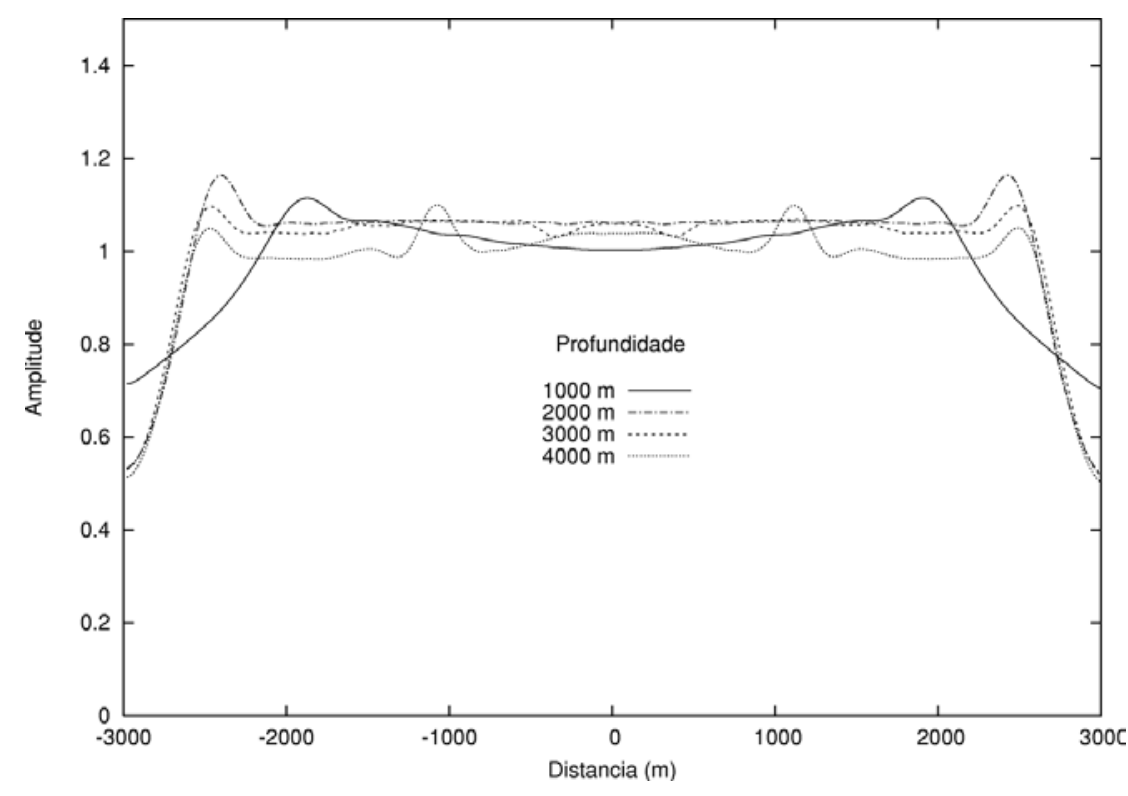

Figura 3 - Amplitudes nos refletores através da migração phase-shift com amplitude verdadeira.

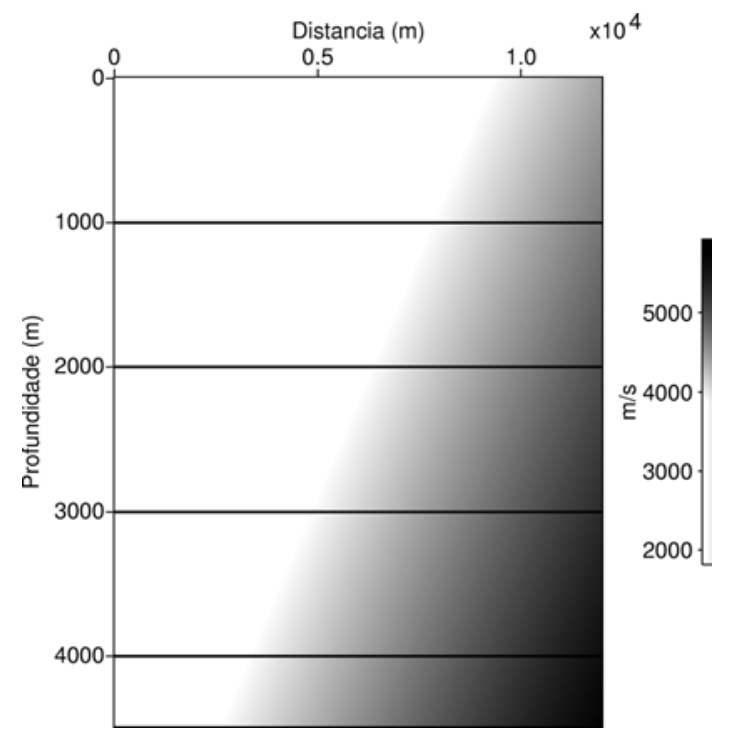

Figura 4 - Campo de velocidade $v(x, z)=2000+0.2 x+0.3 z$ com refletores simulando contrastes de densidade.

métodos PSPI convencional, à esquerda, e PSPI com amplitude verdadeira, à direita.

Da mesma forma que no caso da velocidade variando com a profundidade, a amplitude recuperada nos refletores não deve depender do ângulo nem da profundidade do refletor. Nas Figuras 7 e 8 são apresentadas as amplitudes obtidas nos refletores da Figura 6. Observa-se que o método PSPI com amplitude verdadeira (Figura 8) consegue melhorar substancialmente as amplitudes obtidas no refletor mais raso.

\section{Resultados com o dado Marmousi}

Embora ainda não tenha sido implementada uma condição de imagem para dados com múltiplos tiros, que preserve a amplitude, apresentamos os resultados da migração de dois conjuntos de dados sintéticos acústicos 2D modelados através da equação da onda completa e que por tanto levam em conta de forma correta o espalhamento geométrico de uma fonte linear: 0 dado Sigsbee2A (SMAART JV) e o dado Marmousi (IFP). Mostramos que os métodos implementados PSPI e PSPI-SS com amplitude verda- 


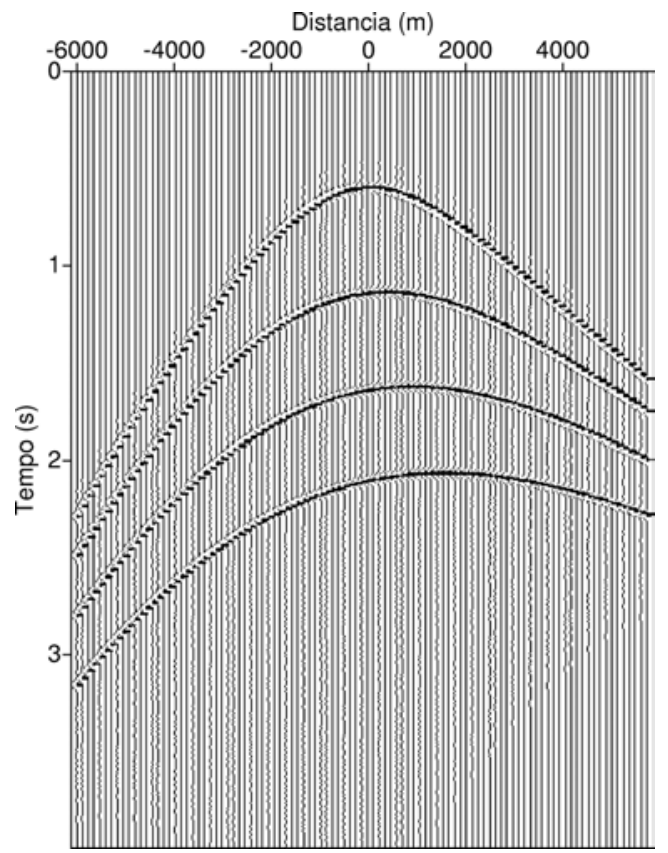

Figura 5 - Dado sintético gerado através de modelamento tipo Kirchhoff.
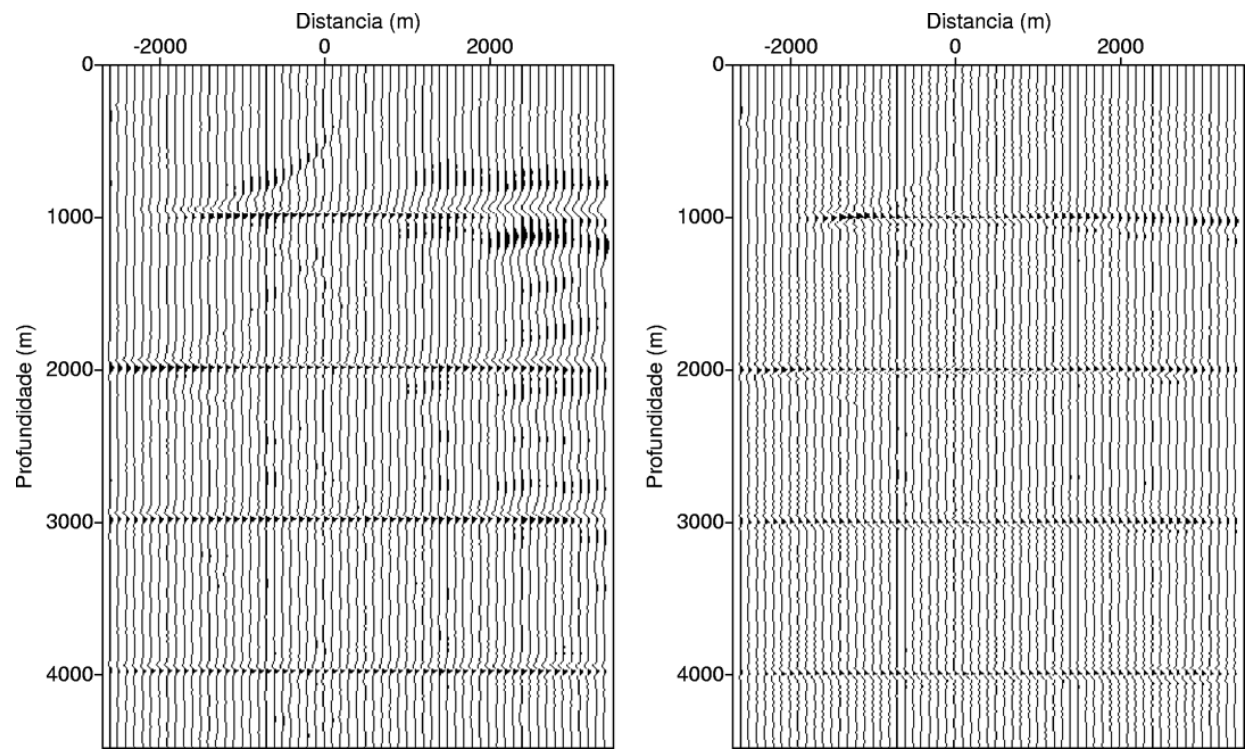

Figura 6 - Imagem obtida através do método PSPI convencional (esquerda). Imagem através do método PSPI com amplitude verdadeira (direita), caso $v(x, z)$

deira e condição de imagem por deconvolução após suavização, melhoram significativamente as amplitudes obtidas em áreas com pobre iluminação.

As Figuras 9, 10 e 11 mostram os resultados da migração PSPI convencional, PSPI com amplitude verdadeira, e PSPI-SS com amplitude verdadeira, respectivamente. Nos resultados das migrações com amplitude verdadeira observa-se uma notável me-
Ihoria das amplitudes e também uma melhor resolução dos refletores profundos.

\section{Resultados com o dado Sigsbee2A}

0 dado sintético acústico Sigsbee2A, modela um conjunto geológico em águas profundas no Golfo de México, ressaltando os problemas de iluminação dos refletores abaixo do corpo de sal, 


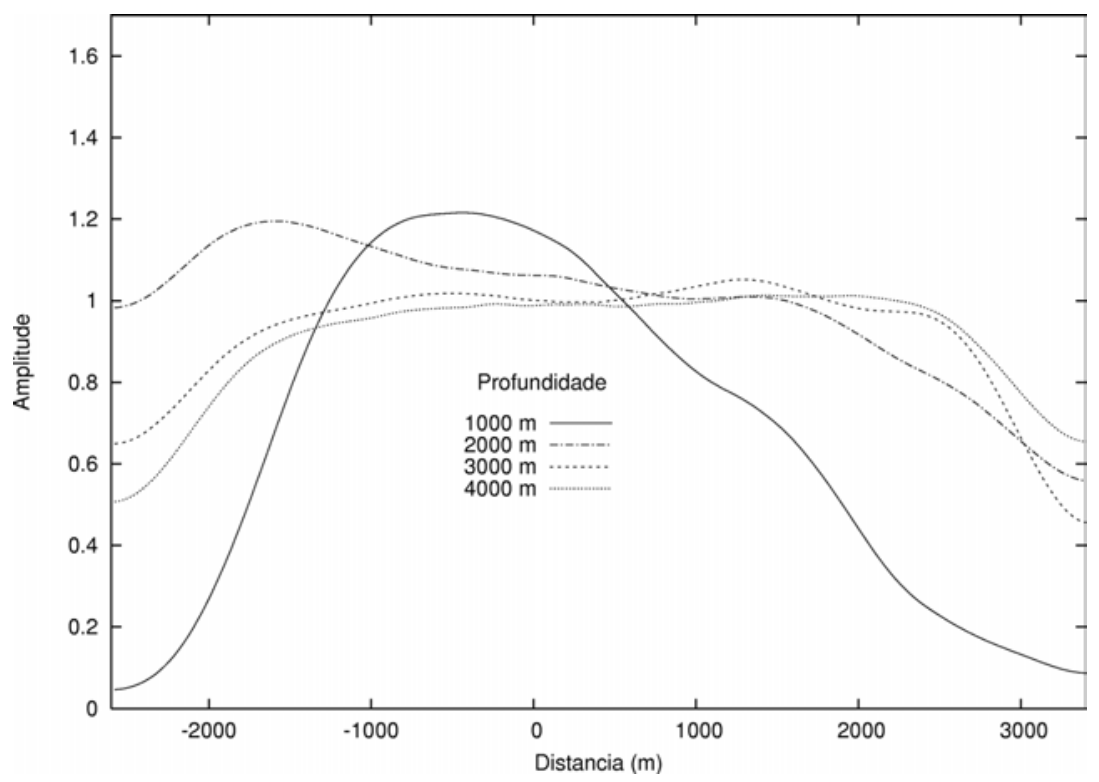

Figura 7 - Amplitudes nos refletores por migração PSPI convencional.

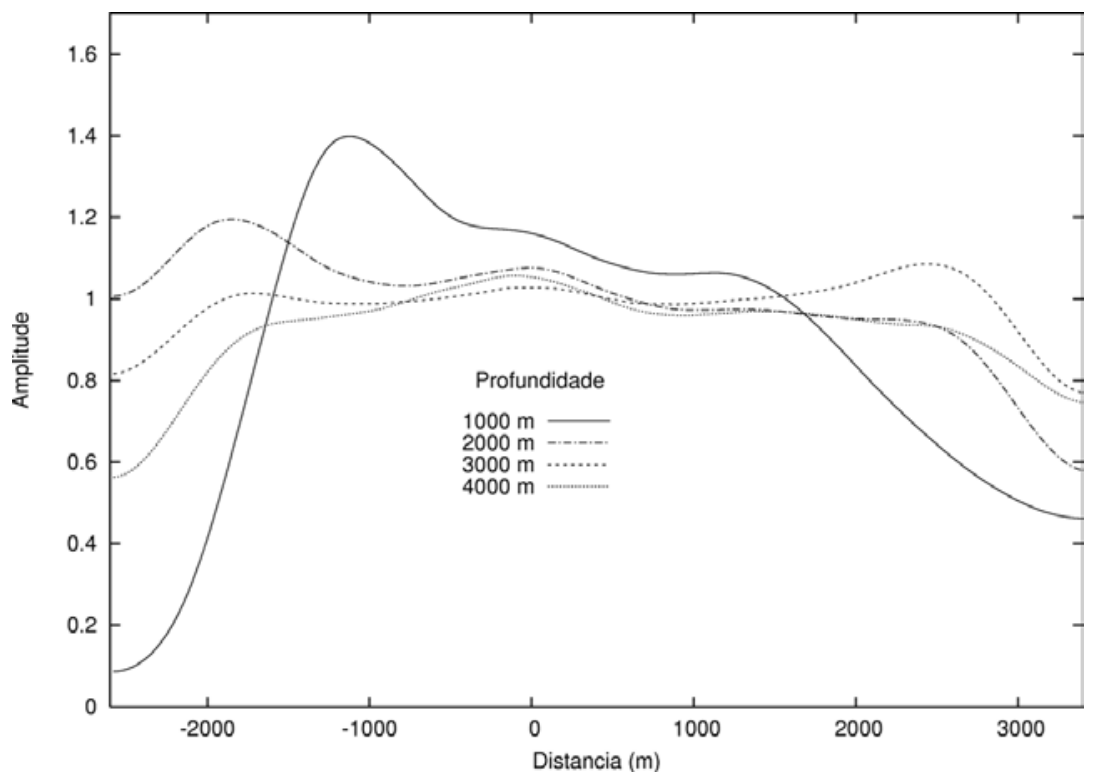

Figura 8 - Amplitudes nos refletores por migração PSPI amplitude verdadeira.

cujo topo é muito irregular. As Figuras 12, 13 e 14 mostram os resultados da migração PSPI convencional, PSPI com amplitude verdadeira, e PSPI-SS amplitude verdadeira, respectivamente, utilizando o macro modelo de velocidades proporcionado para migração em profundidade. Observa-se uma melhor iluminação dos refletores abaixo do corpo de sal com os métodos com amplitude verdadeira, em relação à migração com os operadores convencionais.

\section{CONCLUSÕES}

Os novos operadores unidirecionais com amplitude verdadeira podem ser facilmente implementados em meios que variam com a profundidade através da técnica convencional de migração phaseshift, entretanto sua implementação em meios com variação lateral está ainda em desenvolvimento. As metodologias PSPI com amplitude verdadeira e sua extensão PSPI-SS, propostas neste trabalho, são fáceis de implementar uma vez que os operadores 


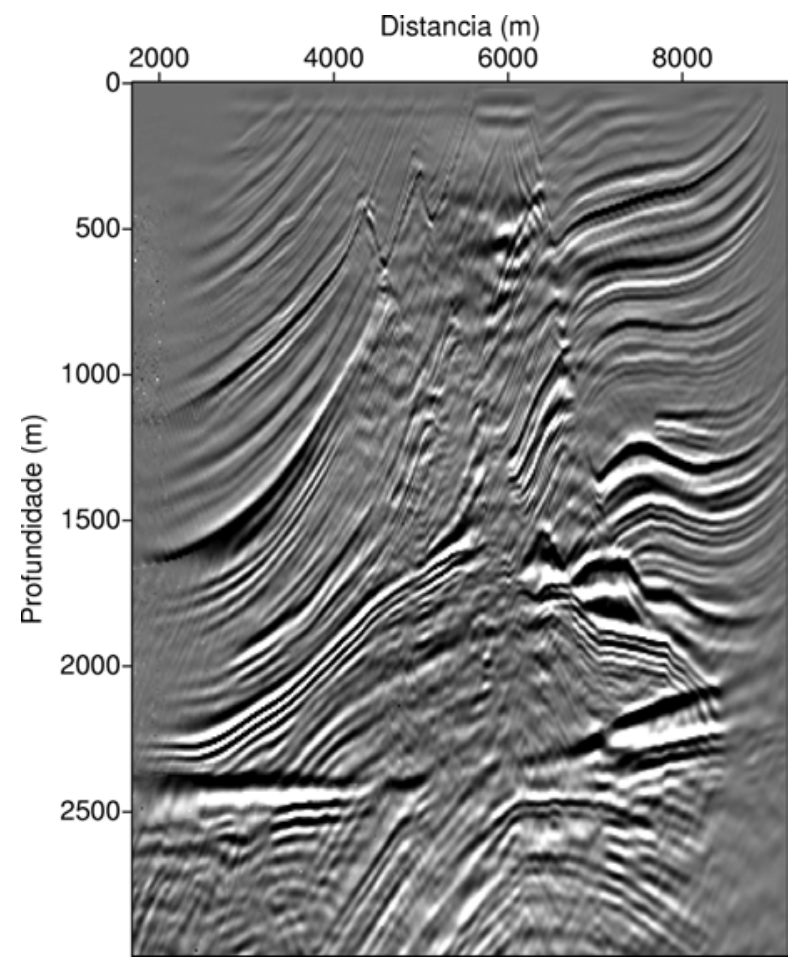

Figura 9 - Imagem migrada com método PSPI convencional.

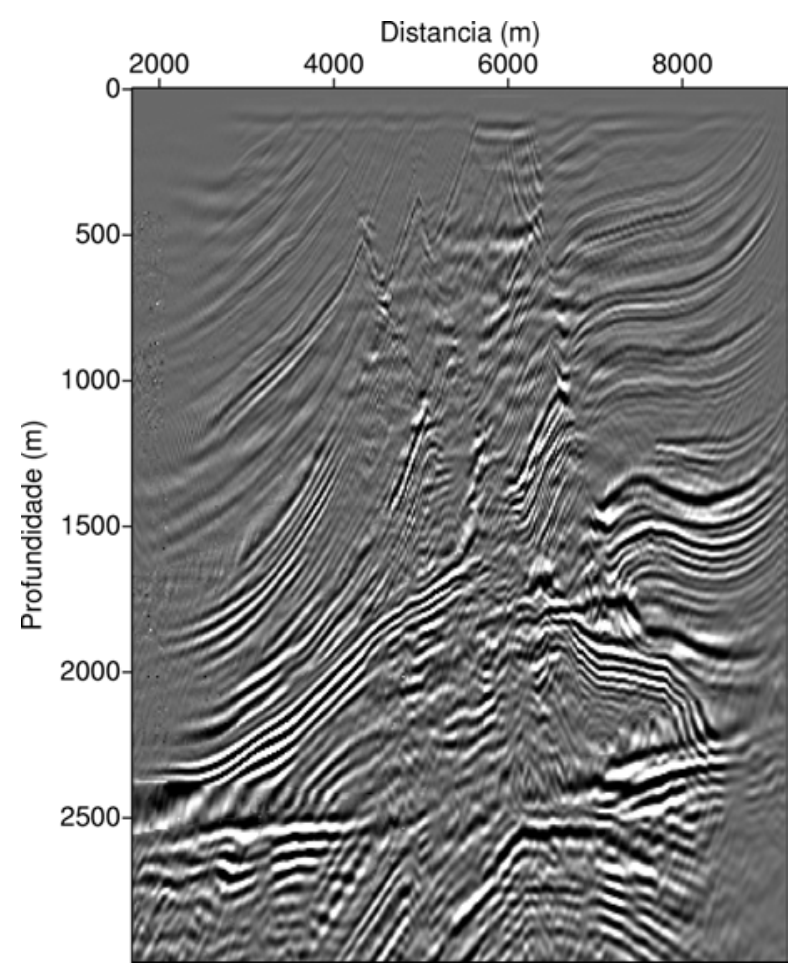

Figura 10 - Imagem migrada com método PSPI com amplitude verdadeira. 
pseudo-diferencias, no caso $v(z)$, se reduzem a multiplicações no domínio de Fourier. Os resultados obtidos tanto no modelo sintético com um único tiro como nos dados sintéticos com múltiplos tiros, mostram melhoria nas amplitudes recuperadas e também uma melhor resolução dos refletores mais profundos através das migrações implementadas baseadas nas equações da onda com amplitude verdadeira.

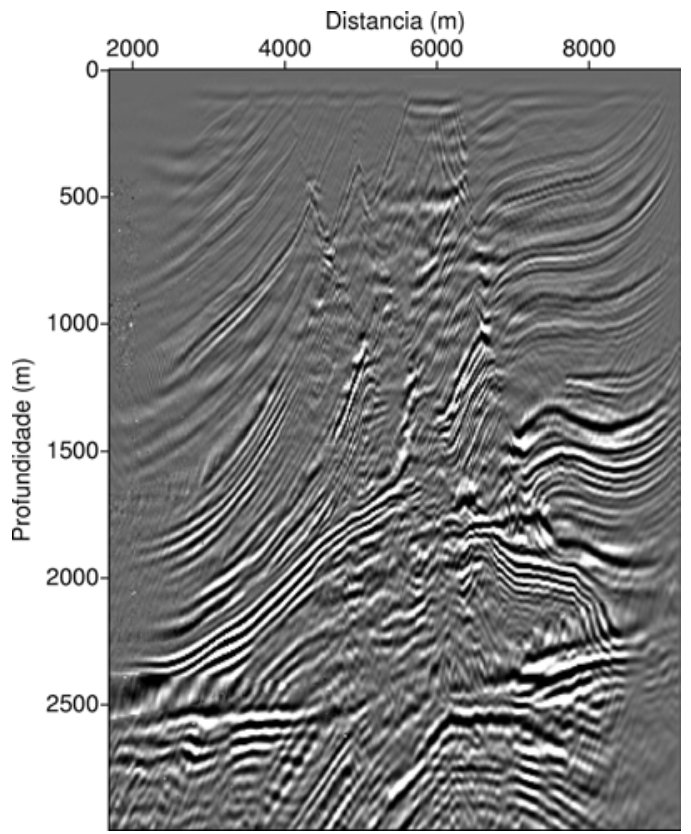

Figura 11 - Imagem migrada com método PSPI-SS com amplitude verdadeira.

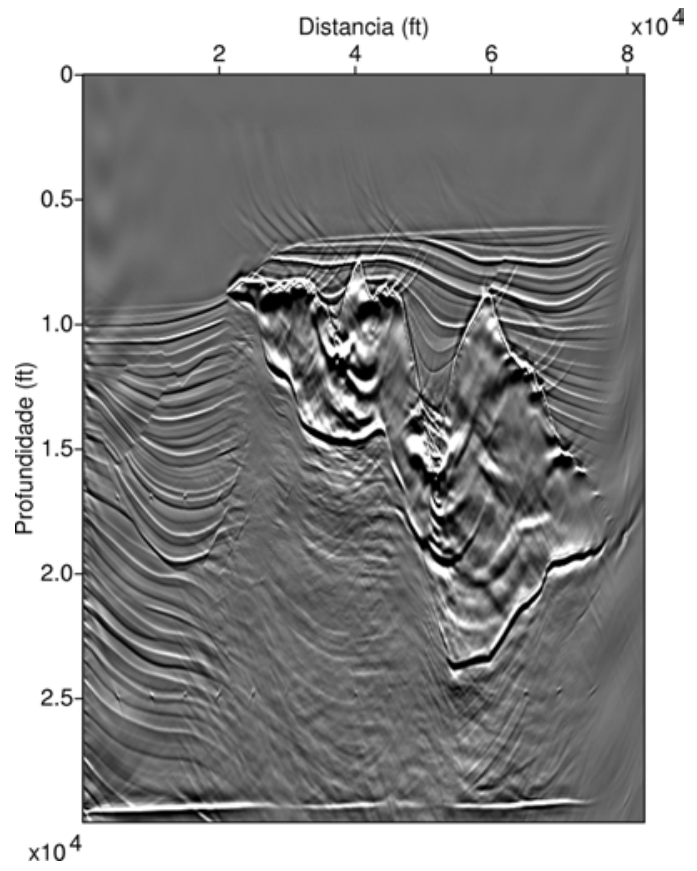

Figura 12 - Imagem migrada com método PSPI convencional.

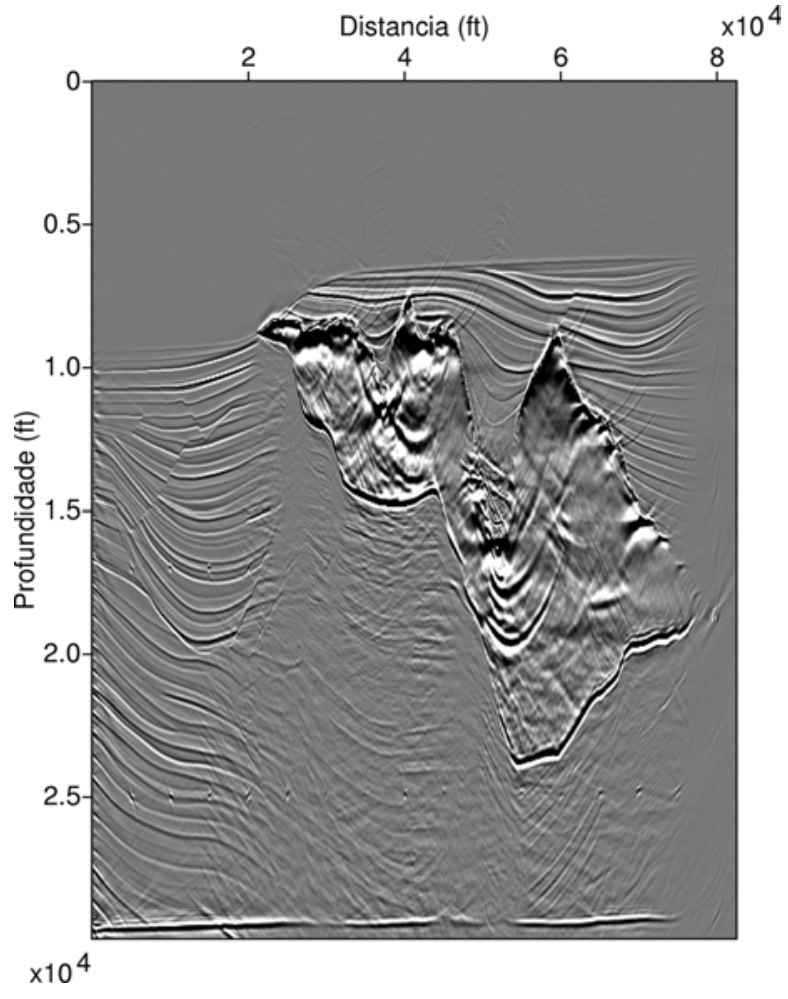

Figura 13 - Imagem migrada com método PSPI com amplitude verdadeira.

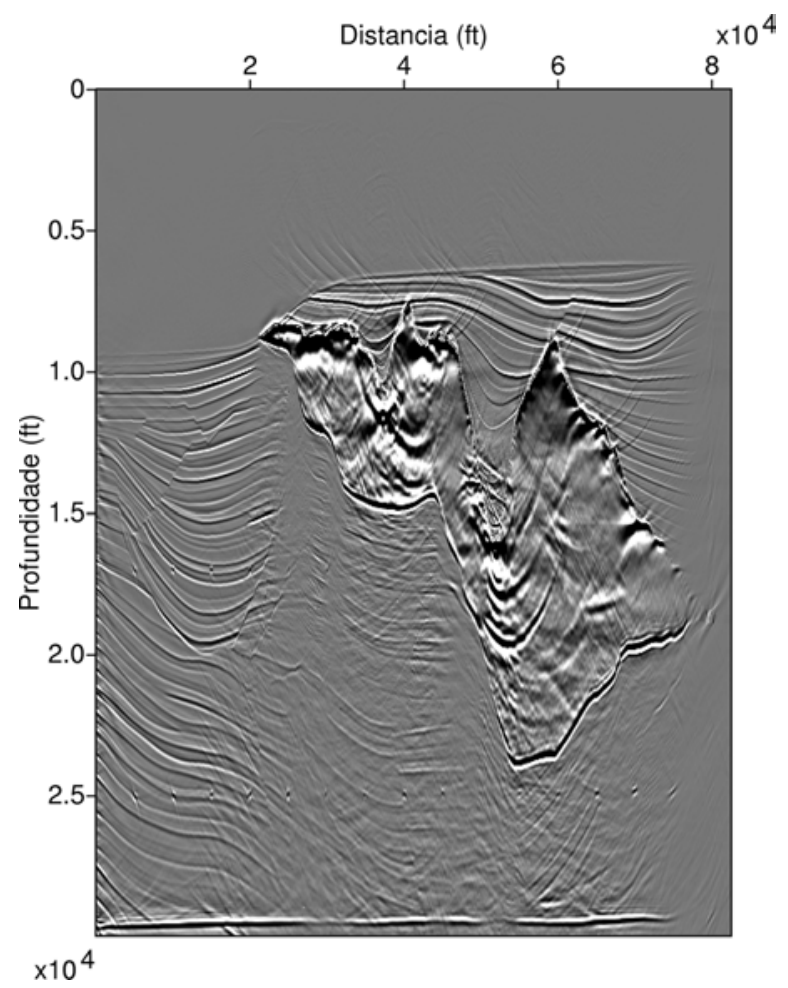

Figura 14 - Imagem migrada com método PSPI-SS com amplitude verdadeira. 


\section{AGRADECIMENTOS}

Os autores agradecem aos revisores pelas valiosas sugestões e comentários, ao LAGEP-CPGG/UFBA, ao CTPETRO/CNPqPetrobras e à FAPESB pelo apoio para o desenvolvimento deste trabalho. Flor A. Vivas agradece também a Universidad de Pamplona (Colômbia) pelo apoio recebido durante o curso de Doutorado em Geofísica no CPGG/UFBA.

\section{REFERÊNCIAS}

ALDUNATE G, PESTANA R \& STOFFA P. 2004. Migração sísmica 2-D pré-empilhamento em profundidade com operadores de extrapolação split-step. Revista Brasileira de Geofísica, 22(2): 153-161.

BAGAINI C, BURKHARD N, ROCCA F \& WYATT K. 1995. Data parallel implementation of 3-D PSPI. 65th Ann. Internat. Mtg., Soc. of Expl. Geophys., Expanded Abstracts, 188-191.

BLEINSTEIN N. 1987. On the imaging of reflectors in the earth. Geophysics, 52: 931-942.

CLAERBOUT J. 1971. Toward a unified theory of reflector mapping. Geophysics, 36: 467-481.

CLAERBOUT J. 1985. Imaging the Earth's interior: Blackwell Scientific Publications, Inc. 398 pp.

COHEN J \& STOCKWELL Jr. 2006. CWP/SU: Seismic Unx Release No. 39: a free package for seismic research and processing, Center for Wave Phenomena, Colorado School of Mines.

GAZDAG J \& SGUAZZERO P. 1984. Migration of seismic data by phase shift plus interpolation. Geophysics, 49: 124-131.
GUITTON A, VALENCIANO A, BEVC D \& CLAERBOUT J. 2006. Robust Illumination Compensation for shot-profile migration. EAGE 68th Conference \& Exhibition, 1-5.

KESSINGER W. 1992. Extended split-step Fourier migration. Ann. Internat. Mtg., Soc. of Expl. Geophys., Expanded Abstracts, 917-920.

RISTOW D \& RÜHL T. 1994. Fourier finite-difference migration. Geophysics, 59: 1882-1893.

SCHULTZ P \& SHERWOOD J. 1980. Depth migration before stack. Geophysics, 45: 376-393.

STOFFA P, FOKKEMA J, de LUNA FREIRE R \& KESSINGER W. 1990. Split-step Fourier migration. Geophysics, 55: 410-421.

VALENCIANO A \& BIONDI B. 2003. 2-D deconvolution imaging condition for shot-profile migration. 73rd Ann. Internat. Mtg., Soc. of Expl. Geophys., Expanded Abstracts, 1059-1062.

WAPENAAR C. 1990. Representation of seismic sources in the one-way wave equations. Geophysics, 55: 786-789.

ZHANG G. 1993. System of coupled equations for upgoing and downgoing waves. Acta Mathematicae Applicatae Sinica, 16(2): 251-263.

ZHANG Y, SUN J, GRAY S, NOTFORS C \& BLEISTEIN N. 2001. Towards accurate amplitudes for one-way wavefield extrapolation of 3-D common shot records. 71st Annual International Meeting (Workshop), SEG. CDROM.

ZHANG Y, ZHANG G \& BLEISTEIN N. 2005. Theory of true-amplitude one-way wave equations and true-amplitude common-shot migration. Geophysics, 70: E1-E10.

\section{NOTAS SOBRE OS AUTORES}

Flor Alba Vivas. Graduada em Física (1997) e mestre em Física, (2001), pela Universidade Industrial de Santander - Colômbia. Atualmente é aluna do curso de Doutorado em Geofísica da Universidade Federal da Bahia - Brasil. É Professora do Departamento de Física da Universidade de Pamplona - Colômbia. Tem atuado em física computacional na área de física de plasma, atualmente sua área de interesse é o processamento e imageamento de dados sísmicos. É membro da SBGf e da SEG.

Reynam C. Pestana. Graduado em Física (UFBA/1983) e Doutor em Geofísica pela UFBA, 1988. Pós-doutorado em Geofísica no Instituto de Geofísica da Universidade de Karlsruhe (Alemanha), 1989-1991, e de 1998-1999 no Instituto de Geofísica da Universidade do Texas em Austin. De 1988 até o presente é pesquisador do CPGG/UFBA, atuando no Grupo de Geofísica de Exploração de Petróleo. Desde 2006 é Professor Associado I do Departamento de Física da Terra e do Meio Ambiente do Instituto de Física e da Pós-graduação em Geofísica da UFBA. Tem atuado no desenvolvimento de métodos e algoritmos de processamento e imageamento sísmicos. Mais recentemente suas pesquisas envolvem técnicas de migração de dados sísmicos decompostos em ondas planas. É membro da SBGf e da SEG. 